\title{
Symmetric Integrable-Polynomial Factorization for Symplectic One-turn-Map Tracking
}

\author{
Jicong Shi ${ }^{1}$ \\ Department of Physics, University of Houston, Houston, Tx77204-5506 \\ Yiton T. Yan \\ SSC Laboratory ${ }^{2}, 2550$ Beckleymeade Ave., Dallas, TX75237
}

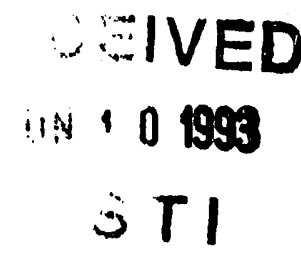

\begin{abstract}
It was found that any homogeneous polynomial can be written as a sum of integrable polynomials of the same degree which Lie transformations can be evaluated exactly. By utilizing symplectic integrators, an integrablepolynomial factorization is developed to convert a symplectic map in the form of Dragt-Finn factorization into a product of Lie transformations associated with integrable polynomials. A small number of factorization bases of integrable polynomials enable one to use high order symplectic integrators so that the high-order spurious terms can be greatly suppressed. A symplectic map can thus be evaluated with desired accuracy.
\end{abstract}

\section{INTRODUCTION}

In large storage rings, high intensity beams are required to circulate for many hours in the presence of nonlinear perturbations of multipole errors in magnets. Extensive computer simulations are thus necessary to investigate the long-term stability of beams. The conventional approach in which trajectories of particles are followed element by element through accelerator structures is, however, very slow in these situations. A substantial computational as well as conceptual simplification is to study the stability of particles by using one-turn maps.

For a large storage ring with thousands of elements, while finding a closed analytical form of one-turn map is impossible, a truncated Taylor expansion of one-turn map-the Taylor map-can be easily obtained. Even though some successes have been reported on using the Taylor maps, the truncation inevitably violates the symplectic nature of systems, and consequently leads to spurious effects if the maps are used to study the long-term stability [1]. A reliable long-term tracking study with the Taylor map is therefore possible only if its nonsymplecticity effect can be eliminated without much reduction in the tracking speed.

In order to eliminate the nonsymplecticity, the Taylor map is usually converted into Lie transformations with

\footnotetext{
'Supported by TNRLC under award FCFY9221 and the U.S. Department of Energy under grant DE-FG05-87ER40374.

2 Operated by the Universities Research Association, Inc., for the U.S. Department of Energy under Contract No, DE-AC3589ER40486.
}

Dragt-Finn factorization [2]. A map in form of Lie transformation is guaranteed to be symplectic, but generally cannot be used for tracking directly because evaluating a nonlinear map in form of Lie transformation is equivalent to solving nonlinear Hamiltonian systems which cannot be done in general. Several methods, such as the jolt factorization [3] and monomial factorization [4], have been proposed to deal with this difficulty by converting Lie transformation from its general the form into special forms that can be evaluated directly. While these methods seem promising, their applications lead to considerable theoretical and computational complexities of which the major difficulty is unpredictability of high order spurious terms that may lead to not enough accurate evaluation of the map.

Since a general Lie transformation corresponds to a nonintegrable system which cannot be evaluated exactly, the challenge here is how to evaluate a Lie transformation approximately without violating the symplecticity and with a controllable accuracy. One way is to divide the nonintegrable system into subsystems which are integrable individually. The set of subsystems of minimum number is the most promising one to serve as the zeroth-order approximation in the sense that it would generate less high order error as well as be a better starting point for higher order treatments. For Lie transformations associated with homogeneous polynomials, we have shown [5] that any polynomial can be written as a sum of integrable polynomials which Lie transformations can be evaluated exactly. Since the number of integrable polynomials can be much smaller than the number of monomials, a factorization based on the integrable polynomials will have much less terms so that a higher order factorization becomes practical. In order to achieve an optimization between a desired accuracy and a fast tracking speed, we have proposed a factorization on the integrable polynomials with symplectic integrators [5]. The advantage of the factorization with symplectic integrators is the suppression of high-order spurious terms to a desired accuracy $[6-8]$.

\section{INTEGRABLE POLYNOMIAL IN LIE TRANSFORMATION}

A polynomial in $\vec{z}$ is called integrable polynomial if its associated Hamiltonian system is integrable, i.e. its asso- 


\section{DISCLAIMER}

This report was prepared as an account of work sponsored by an agency of the United States Government. Neither the United States Government nor any agency thereof, nor any of their employees, makes any warranty, express or implied, or assumes any legal liability or responsibility for the accuracy, completeness, or usefulness of any information, apparatus, product, or process disclosed, or represents that its use would not infringe privately owned rights. Reference herein to any specific commercial product, process, or service by trade name, trademark, manufacturer, or otherwise does not necessarily constitute or imply its endorsement, recommendation, or favoring by the United States Government or any agency thereof. The views and opinions of authors expressed herein do not necessarily state or reflect those of the United States Government or any agency thereof. 
ciated Lie transformation can be evaluated exactly. Let $\left\{g_{i}^{(k)} \mid k=1,2, \ldots, N_{g}\right\}$ denote a set of integrable polynomials of degree $i$. In what follows, we shall show that any polynomial in $\vec{z}$ can be expressed as a sum of integrable polynomials of the same degree, i.e.

$$
f_{i}(\vec{z})=\sum_{\left(\sum \sigma,\right)=i} a(\vec{\sigma}) z_{1}^{\sigma_{1}} p_{1}^{\sigma_{2}} z_{2}^{\sigma_{3}} p_{2}^{\sigma_{4}} z_{3}^{\sigma_{s}} p_{3}^{\sigma_{\beta}}=\sum_{k=1}^{N_{g}} g_{i}^{(k)}
$$

where $f_{i}$ is any homogeneous polynomial of degree $i$ in phase-space vector $\vec{z}=\left(z_{1}, p_{1}, z_{2}, p_{2}, z_{3}, p_{3}\right)$ and $a(\vec{\sigma}) \mathrm{s}$ are constant coefficients. After factorizing it as a product of Lie transformations associated with integrable polynomials, $\exp \left(: f_{i}:\right) \vec{z}$ can be therefore evaluated directly. Since the minimum number of integrable polynomials $N_{g}$ is much smaller than the number of monomials, the accuracy of factorization with $\left\{g_{i}^{(k)}\right\}$ as bases can be carried to a desired order with the use of symplectic integrators.

Homogeneous polynomials of degree 3 iri 6 -variables consist of 56 monomials which can be grouped under 8 integrable polynomials of degree $3,\left\{g_{3}^{(n)} \mid n=1,2, \ldots, 8\right\}$ :

$$
\begin{aligned}
g_{3}^{(1)}= & c_{1}^{(1)} z_{1}^{3}+c_{2}^{(1)} z_{1}^{2} p_{1}+c_{3}^{(1)} z_{2}^{3} \\
& +c_{4}^{(1)} z_{2}^{2} p_{2}+c_{5}^{(1)} z_{3}^{3}+c_{6}^{(1)} z_{3}^{2} p_{3} \\
g_{3}^{(2)}= & c_{1}^{(2)} p_{1}^{3}+c_{2}^{(2)} p_{1}^{2} z_{1}+c_{3}^{(2)} p_{2}^{3} \\
& +c_{4}^{(2)} p_{2}^{2} z_{2}+c_{5}^{(2)} p_{3}^{3}+c_{6}^{(2)} p_{3}^{2} z_{3} \\
g_{3}^{(2+i)}= & z_{i} h_{2}^{(2+i)}\left(z_{j}, p_{j}, z_{k}, p_{k}\right) \\
g_{3}^{(5+i)}= & p_{i} h_{2}^{(5+i)}\left(z_{j}, p_{j}, z_{k}, p_{k}\right)
\end{aligned}
$$

where $(i, j, k)$ goes over all cyclic permutations of $(1,2,3)$. $h_{2}^{(n)} \mathrm{s}$ are homogeneous polynomials of degree 2 in 4 variables. $c_{m}^{(n)}$ is the coefficient of the corresponding monomial in $f_{3}$. It should be noted that the decomposition of $f_{i}$ into integrable polynomials is not unique. $g_{3}^{(1)}$ and $g_{3}^{(2)}$ can be further combined into a single integrable polynomial since the Hamiltonian system with $H=-\left(g_{3}^{(1)}+g_{3}^{(2)}\right)$ is integrable. We chose two separate integrable polynomials instead of the combined one because the solution for the combined one cannot be written in a closed form and directly used in tracking.

The Lie transformations associated with integrable polynomials can be converted into simple iterations [5]:

$$
\begin{aligned}
& e^{g_{3}^{(1)}:} z_{i}=\frac{z_{i}}{1+c_{2 i}^{(1)} z_{i}} \\
& e^{: g_{3}^{(1)}:} p_{i}=-\frac{\left(c_{2 i-1}^{(1)} z_{i}+c_{2 i}^{(1)} p_{i}\right)\left(c_{2 i}^{(1)} z_{i}+1\right)^{3}+c_{2 i-1}^{(1)} z_{i}}{c_{2 i}^{(1)}\left(1+c_{2 i}^{(1)} z_{i}\right)} \\
& e^{: g_{3}^{(2)}:} z_{i}=\frac{\left(c_{2 i-1}^{(2)} p_{i}+c_{2 i}^{(2)} p_{i}\right)\left(c_{2 i}^{(2)} p_{i}-1\right)^{3}-c_{2 i-1}^{(2)} p_{i}}{c_{2 i}^{(2)}\left(1-c_{2 i}^{(2)} p_{i}\right)} \\
& e^{: g_{3}^{(2)}}: p_{i}=\frac{p_{i}}{1-c_{2 i}^{(2)} p_{i}}
\end{aligned}
$$

$$
\begin{aligned}
e^{: g_{3}^{(2+i)}:} z_{i} & =z_{i} \\
e^{: g_{3}^{(2+i)}}: p_{i} & =p_{i}+h_{2}^{(2+i)}\left(z_{j}, p_{j}, z_{k}, p_{k}\right), \\
e^{: g_{3}^{(5+i)}}: z_{i} & =z_{i}-h_{2}^{(5+i)}\left(z_{j}, p_{j}, z_{k}, p_{k}\right), \\
e^{: g_{3}^{(8+i)}}: p_{i} & =p_{i} \\
e^{: g_{3}^{(2+i)}}: \vec{r} & =U_{2+i}^{-1} \exp \left(z_{i} \Lambda_{2+i}\right) U_{2+i} \vec{r} \\
e^{: g_{3}^{(5+i)}}: \vec{r} & =U_{5+i}^{-1} \exp \left(p_{i} \Lambda_{5+i}\right) U_{2+i} \vec{r}
\end{aligned}
$$

where $(i, j, k)$ goes over all cyclic permutations of $(1,2,3)$.

$$
\begin{gathered}
\vec{r}=\left(\begin{array}{llll}
z_{j} & p_{j} & z_{k} & p_{k}
\end{array}\right)^{T} \\
\frac{\partial}{\partial \vec{r}}=\left(\begin{array}{llll}
\frac{\partial}{\partial z_{j}} & \frac{\partial}{\partial p_{j}} & \frac{\partial}{\partial z_{k}} & \frac{\partial}{\partial p_{k}}
\end{array}\right)^{T}, \\
\Lambda_{n}=U_{n}\left[\begin{array}{ccc}
\Gamma \frac{\partial}{\partial \vec{r}} & \left(\frac{\partial}{\partial \vec{r}}\right)^{T} & h_{l}^{(n)}
\end{array}\right] U_{n}^{-1} \\
=\left(\begin{array}{cccc}
\lambda_{1}^{(n)} & 0 & 0 & 0 \\
0 & \lambda_{2}^{(n)} & 0 & 0 \\
0 & 0 & \lambda_{3}^{(n)} & 0 \\
0 & 0 & 0 & \lambda_{4}^{(n)}
\end{array}\right),
\end{gathered}
$$

where superscript $T$ denotes the transpose and $\Gamma$ is a 4 dimensional antisymmetric matrix:

$$
\Gamma=\left(\begin{array}{cccc}
0 & -1 & 0 & 0 \\
1 & 0 & 0 & 0 \\
0 & 0 & 0 & -1 \\
0 & 0 & 1 & 0
\end{array}\right)
$$

Similarly, 126, 252, and 462 monomials of homogeneous polynomials of degree 4,5 , and 6 in 6 -variables can be grouped into 20,42 , and 79 iniegrable polynomials of degree 4,5 , and 6 respectively [5].

\section{SYMMETRIC \\ INTEGRABLE-POLYNOMIAL FACTORIZATION}

With integrable polynomials, a symplectic map in the form of the Dragt-Finn factorization can be rewritten as

$$
\vec{U}_{s}(\vec{z})=R \prod_{i=3} \exp \left(\sum_{n=1}^{N_{i}}: g_{i}^{(n)}:\right) \vec{z}
$$

where $R$ denotes the linear transformation and $N_{i}$ is the number of integrable polynomials of degree $i$. By means of Campbell-Baker-Hausdorff ( $\mathrm{CBH}$ ) formula [2], one can, in principle, convert the Lie transformation associated with a sum of integrable polynomials into a product of Lie transformations associated with integrable polynomials. The separation of low-order $\exp \left(: g_{i}^{(n)}:\right)$, however, generates high-order spurious terms which may cascade so large that the truncation becomes invalid. Moreover, it is unclear which integrable polynomial of the same degree should be 
arranged in precedence of the others in the series of Lie transformations. Such nonsymmetric property actually affects the accuracy of the map by generating larger highorder spurious terms.

In construction of symplectic integrators for numerical integration, techniques have been developed to formulate a Lie transformation for a sum of Lie operators by Lie transformations for individual Lie operators with a controllable truncation error [6-8]. It was shown [8] that all even-order symplectic integrators can be systematically constructed in a symmetric form. The symmetric feature greatly suppresses the high-order truncation error. A small number of factorization bases with integrable polynomials enable us to utilize these symplectic integrators.

For $i \geq 5$, since $\left(: g_{i}^{\left(n_{1}\right)}: g_{i}^{\left(n_{2}\right)}\right)$ is a homogenous polynomial with degree higher than 7 , a factorization with up to the 7 th order is easily obtained by directly using the first-order integrator

$$
\exp \left(\sum_{n=1}^{N_{i}}: g_{i}^{(n)}:\right)=\prod_{n=1}^{N_{i}} \exp \left(: g_{i}^{(n)}:\right)+\epsilon(2 i-2),
$$

where $i \geq 5 . \epsilon(2 i-2)$ represents the truncated terms which are homogeneous polynomial with degree higher than $2 i-$ 3 . For $i=5$ and 6 , the lowest-order truncated term is homogeneous polynomial of degree 8 and 10 respectively.

For homogeneous polynomials of degree 4 , we use the 2nd-order integrator and obtain a 7 th-order symplectic map

$$
\exp \left(\sum_{n=1}^{20}: g_{4}^{(n)}:\right)=\left(\prod_{i=1}^{20} e^{: \frac{1}{2} g_{4}^{\left(n_{i}\right)}}\right) \prod_{i=1}^{20} e^{i \frac{1}{2} g_{4}^{\left(21-n_{i}\right)}}+\epsilon(8)
$$

where $\left(n_{1}, n_{2}, \ldots, n_{20}\right)$ is any permutation of $(1,2, \ldots, 20)$. The lowest-order truncated term in Eq. (20) is a homogeneous polynomial of degree 8 .

In order to obtain a 6 th-order symplectic map, we has to use the 4 th-order integrator $[6-8]$ to factorize $\exp \left(: f_{3}:\right)$, which yields a product of $7^{3}=343 \mathrm{Lie}$ transformations associated with integrable polynomials:

$$
\begin{gathered}
\exp \left(\sum_{n=1}^{8}: g_{3}^{(n)}:\right)=\prod_{i=1}^{7} \prod_{j=1}^{7} \prod_{k=1}^{7} \exp \left(: d_{i} d_{j} d_{k} D_{i j k}:\right)+\epsilon(7) . \\
d_{1}=d_{7}=\frac{1}{2\left(2-2^{1 / 3}\right)}, \quad d_{2}=d_{6}=\frac{1}{2-2^{1 / 3}}, \\
d_{3}=d_{5}=\frac{1-2^{1 / 3}}{2\left(2-2^{1 / 3}\right)}, \quad d_{4}=\frac{-2^{1 / 3}}{2-2^{1 / 3}} .
\end{gathered}
$$

$D_{i j k}$ is a integrable polynomial of degree 3 which can be chosen according to following pattern

$$
i=\text { even }\left\{\begin{array}{c}
j=\text { even } \begin{cases}k=\text { even, } & D_{i j k}=g_{3}^{\left(n_{1}\right)} \\
k=\text { odd, }, & D_{i j k}=g_{3}^{\left(n_{2}\right)}\end{cases} \\
j=\text { odd } \begin{cases}k=\text { even, } & D_{i j k}=g_{3}^{\left(n_{3}\right)} \\
k=\text { odd, } & D_{i j k}=g_{3}^{\left(n_{4}\right)}\end{cases}
\end{array}\right.
$$

$$
i=\text { odd }\left\{\begin{array}{c}
j=\text { even } \begin{cases}k=\text { even, } & D_{i j k}=g_{3}^{\left(n_{\mathrm{B}}\right)} \\
k=\text { odd }, & D_{i j k}=g_{3}^{\left(n_{8}\right)}\end{cases} \\
j=\text { odd } \begin{cases}k=\text { even, } & D_{i j k}=g_{3}^{\left(n_{7}\right)} \\
k=\text { odd, } & D_{i j k}=g_{3}^{\left(n_{8}\right)}\end{cases}
\end{array}\right.
$$

where $\left(n_{1}, n_{2}, n_{3}, n_{4}, n_{5}, n_{6}, n_{7}, n_{8}\right)$ is any permutation of the first eight digits, $(1,2,3,4,5,6,7,8)$. The lowestorder truncated term in Eq. (21) consists of homogeneous polynomials of degree 7 .

\section{Conclusion}

We have shown that any polynomial can be written as a sum of integrable polynomials of the same degree. The number of optimized integrable polynomials is much smaller than the number of monomials. For homogeneous polynomials of degree 3 , to 6 , we were able to group $56,126,252$, and 462 monomials into $8,20,42$, and 79 integrable polynomials respectively. All Lie transformations associated with these integrable polynomials were translated into simple iterations which can be directly used in tracking. By utilizing the symmetric symplecticintegrators, we have developed a factorization scheme based on the integrable polynomials in which Lie transformations associated with homogeneous polynomials are converted into a product of Lie transformations associaled with integrable polynomials. A much smaller number of integrable polynomials not only serves a more accurate set of factorization bases but also enable us to use high order factorization schemes so that the truncation error can be greatly suppressed. The map in the form of Lie transformations associated with integrable polynomials could therefore be a reliable model for studying the long-term behavior of symplectic systems in the phase space region of interest.

\section{References}

[1 ] Y. T. Yan, AIP Conf. Proc. 249, ed. M. Month, (AIP, 1992), p.378, and references therein.

[2 ] A. Dragt and J. Finn, J. Math. Phys. 17, 2215 (1976).

[3 ] J. Irwin, SSCL Report SSCL-228, (1989); A. Dragt, I. Gjaja, and G. Rangarajan, Proc. IEEE Accel. Conf., p. 1621 (1991).

[4] I. Gjaja, preprint, (1992).

[5 ] J. Shi and Y. T. Yan, preprint, (1993).

[6] E. Forest and R. D. Ruth, Physica D 43, 105 (1990).

[7 ] F. Neri, Lie algebras and canonical integration, Dept. of Phys., Univ. of Maryland, preprint (1988).

[8 ] H. Yoshida, Phys. Lett. 150, 262 (1990). 

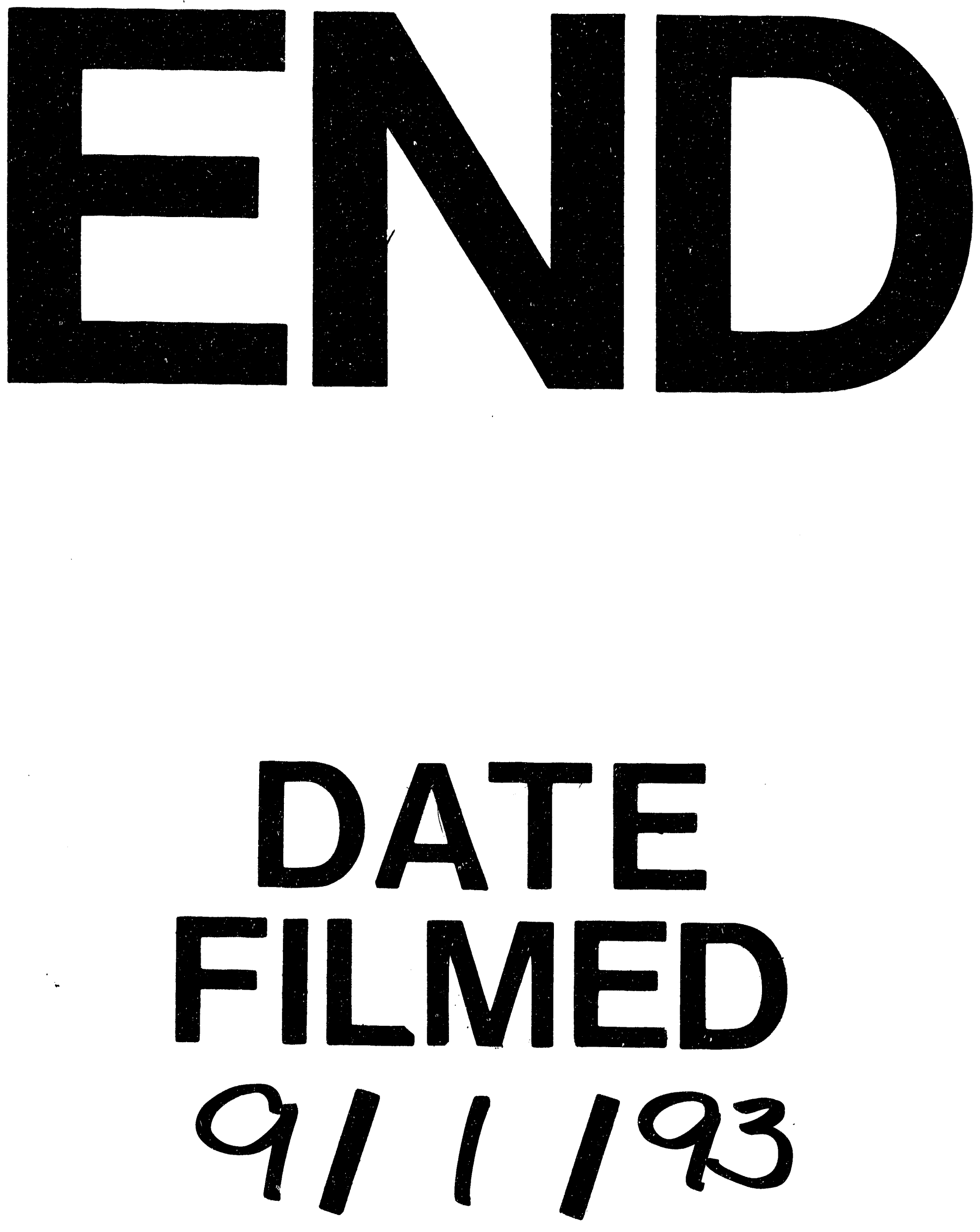
